\title{
Cosmic-ray variability on the multi-millennial time scale: A new multi-proxy reconstruction
}

\author{
llya Usoskin* \\ University of Oulu, Finland \\ E-mail: ilya.usoskindoulu.fi

\section{Chi Ju Wu and Natalie Krivova and Sami K. Solanki \\ Max-Planck-Institut für Sonnensystemforschung, Göttingen, Germany}

\section{Gennady Kovaltsov}

Ioffe Physical-Technical Institute, St. Petersburg, Russia

\section{Meanié Baroni and Edouard Bard}

CEREGE, Aix-Marseille Université, CNRS, NRD, INRA, Collège de France, Technopôle de

l'Arbois, Aix-en-Provence, France

On the time scale of up to millennia the flux of cosmic rays outside the Heliosphere can be assumed roughly constant, and the cosmic-ray variability observed near Earth is driven by solar magnetic activity. Thus, using data on cosmogenic isotopes measured in natural terrestrial archives, past solar activity can be reconstructed. The most important cosmogenic isotopes are radiocarbon ${ }^{14} \mathrm{C}$ and beryllium ${ }^{10} \mathrm{Be}$. However, because of the diversity of the proxy archives, it is difficult to build a homogeneous reconstruction, and previous studies showed inconsistencies with each other. Here we report a new consistent multi-proxy reconstruction of the cosmic-ray variability over the Holocene (last 9000 years), using all available long-span datasets of ${ }^{10} \mathrm{Be}$ and ${ }^{14} \mathrm{C}$ in terrestrial archives ( $\mathrm{six}{ }^{10} \mathrm{Be}$ series of different ice cores from Greenland and Antarctica, as well as the global ${ }^{14} \mathrm{C}$ production series). We have applied a new method, based on a Bayesian approach, which yields the most probable values of the solar modulation as well as straightforward estimates of the related uncertainties. The final reconstruction indicates the presence of a slow 6-7 millennia 'wave' in the long-term evolution of solar activity, with lows at ca. 5500 BC and $1000 \mathrm{AD}$. Two distinct components of solar activity were confirmed: the main component, corresponding to the "normal" moderate level, and a component corresponding to grand minima. A possible existence of a component representing grand maxima cannot be separated from the main component in a statistically significant manner.

36th International Cosmic Ray Conference -ICRC2019-

July 24th - August 1st, 2019

Madison, WI, U.S.A.

\footnotetext{
*Speaker.
} 


\section{Introduction}

The flux of cosmic rays may be considered as roughly constant and isotropic outside of the heliosphere on the time scales of up to tens of millennia, owing to the diffusive transport of cosmic rays in the Galaxy. However, the flux of low-energy $(<50 \mathrm{GeV})$ cosmic rays is highly variable in the vicinity of Earth, that is know as cosmic-ray modulation. Thus, cosmic-ray variability provides a potential tool to investigate solar/heliospheric physics in the past long before any direct measurements became available [피. Two processes define the cosmic-ray modulation near Earth: solar magnetic activity, and the geomagnetic-field shielding.

The heliospheric modulation changes the flux of incoming cosmic rays in the heliosphere via aggregate of four main processes affecting cosmic-ray particles: scattering on magnetic-field inhomogeneties, convection and adiabatic cooling in the radially expanding solar wind and drifts [44]]. Geomagnetic filed, which shields Earth from incoming charged particles depending on their energy/rigidity, changes slowly, and normally its variability is neglected on short time scales, but for the longer time scales it may become dominant and must be considered. Correction for the changing geomagnetic field is straightforward once it is known independently, e.g., from paleo/archeomagnetic data [2]].

Variability of the flux of cosmic rays is recorded in natural, independently dateable archives, such as tree trunks, ice cores or sediments, where cosmogenic isotopes are preserved. Cosmogenic isotopes are called so because their only (or the main) source at Earth is related to cosmic-ray induced nuclear reactions in the Earth's atmosphere [䧃]. Upon production, they follow a transport and deposition/storage in the terrestrial system before being recorded in the archive. A very useful isotopes is radiocarbon $\left({ }^{14} \mathrm{C}\right)$ produced as a product $n p$-reaction (called 'neutron capture') on atmospheric nitrogen, oxidized to ${ }^{14} \mathrm{CO}_{2}$ and stored, after taking part in the global carbon cycle, in tree rings, corals etc. Another useful isotope is beryllium- $10{ }^{10} \mathrm{Be}$ which is produced as a result of spallation of atmospheric oxygen and nitrogen by primary and secondary particles of the acosmicray induced atmospheric cascade. After production it is believed to be attached to be attached to aerosols and quickly (within 1-2 years) precipitate. It is typically measured in polar (Greenland or Antarctic) ice cores.

Accordingly, by measuring the content of cosmogenic isotopes in such natural archives, one can assess the cosmic-ray variability and hence the heliospheric modulation, ultimately defined by solar magnetic activity, in the past on the time scale of up to twelve millennia, the Holocene. To do it quantitatively, one needs several components:

- Precise and independently dated measurements of cosmogenic isotopes;

- Independently know geomagnetic field intensity;

- Quantitative model of the isotope production in the atmosphere;

- Model of transport and deposition of the isotopes in the terrestrial system.

Significant progress have been made recently in all these components leading to different recon-

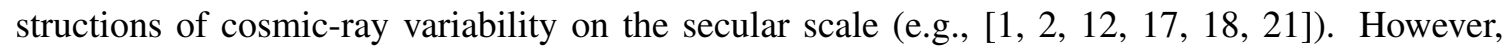
most of the previous reconstructions were based on individual isotope records. Even in case of 
Table 1: Data series used in this work to reconstruct solar activity.

\begin{tabular}{|c|c|c|c|c|}
\hline Isotope & Series & Period (-BC/AD) & Resolution & Reference \\
\hline${ }^{14} \mathrm{C}$ & IntCal (Global) & $-8000-1950$ & $10-\mathrm{yr}$ & [एव] \\
\hline${ }^{10} \mathrm{Be}$ & GRIP (Greenland) & $-7375-1645$ & $10-\mathrm{yr}$ & [II], 25, 27] \\
\hline${ }^{10} \mathrm{Be}$ & EDML (Antarctica) & $-7440-730$ & $10-\mathrm{yr}$ & [ए8] \\
\hline${ }^{10} \mathrm{Be}$ & NGRIP (Greenland) & $1389-1994$ & $1-\mathrm{yr}$ & [[]] \\
\hline${ }^{10} \mathrm{Be}$ & Dye3 (Greenland) & $1424-1985$ & $1-\mathrm{yr}$ & [3], प0] \\
\hline${ }^{10} \mathrm{Be}$ & Dome Fuji (Antarctica) & $690-1880$ & $\sim 5-\mathrm{yr}$ & [] \\
\hline${ }^{10} \mathrm{Be}$ & South Pole (Antarctica) & $850-1960$ & $10-\mathrm{yr}$ & [D, प5] \\
\hline
\end{tabular}

multi-proxy studies, reconstructions were based on individual records and then simply compared

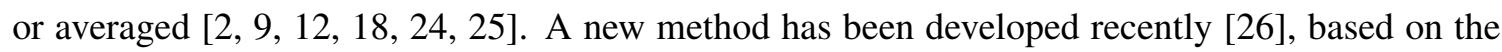
Bayesian approach to find the most probably evolution of solar activity and its uncertainties. Here we apply this method to available datasets and reconstruct solar modulation of cosmic rays on the multi-millennial time scale.

\section{Data sets}

We used seven data series as listed in Table $\mathbb{~}$ : one global ${ }^{14} \mathrm{C}$ dataset and six regional ${ }^{10} \mathrm{Be}$ series, covering different time intervals between $8000 \mathrm{BC}$ and $1950 \mathrm{AD}$. For consistency, all datasets were resampled to the decadal time resolution, before the analysis. Since only ${ }^{14} \mathrm{C}$ has the ' $\mathrm{ab}$ solute' dating based on dendrochronology, while ice-cores may have a 'floated', by up to a few decades, chronology [四]. Accordingly, a wiggle-matching was applied to ${ }^{10} \mathrm{Be}$ series to 'tie' them to the tree-ring scale, using the method of [26].

As the geomagnetic data, we used a range of ensemble paleomagnetic reconstruction, using the model GMAG.9k of the virtual axial dipole moment (VADM) covering all known uncertainties, as described in [R]], that covers the period since $6700 \mathrm{BC}$.

\section{Solar modulation by Bayesian approach}

Solar modulation of cosmic rays is usually quantified via the modulation potential $\phi$ (in units of rigidity, MV), which does not have a clear physical meaning but provides a very handy singleparameter description of the cosmic-ray spectrum near Earth [6, [0]]. A typical way to reconstruct the modulation potential is via inverting the problem: from the measured/estimated production rate of an isotope, $Q(t)$, the value of $\phi(t)$ is assessed for any time moment $t$ by applying a isotope production model and the independently known geomagnetic field model $M(t)$. This however leads to a discrepancy between the results obtained from individual series [ $[8,25]$. These individual series can be averaged, linearly or non-linearly (cf. [ए8]), but this does not take into account the uncertainties of the data and models.

Here we use another approach, based on Bayesian methodology, as developed recently [26]. The method is based on the finding the most probable value of $\phi$ and its uncertainties which matches all the data for given time $t$, as follows. 


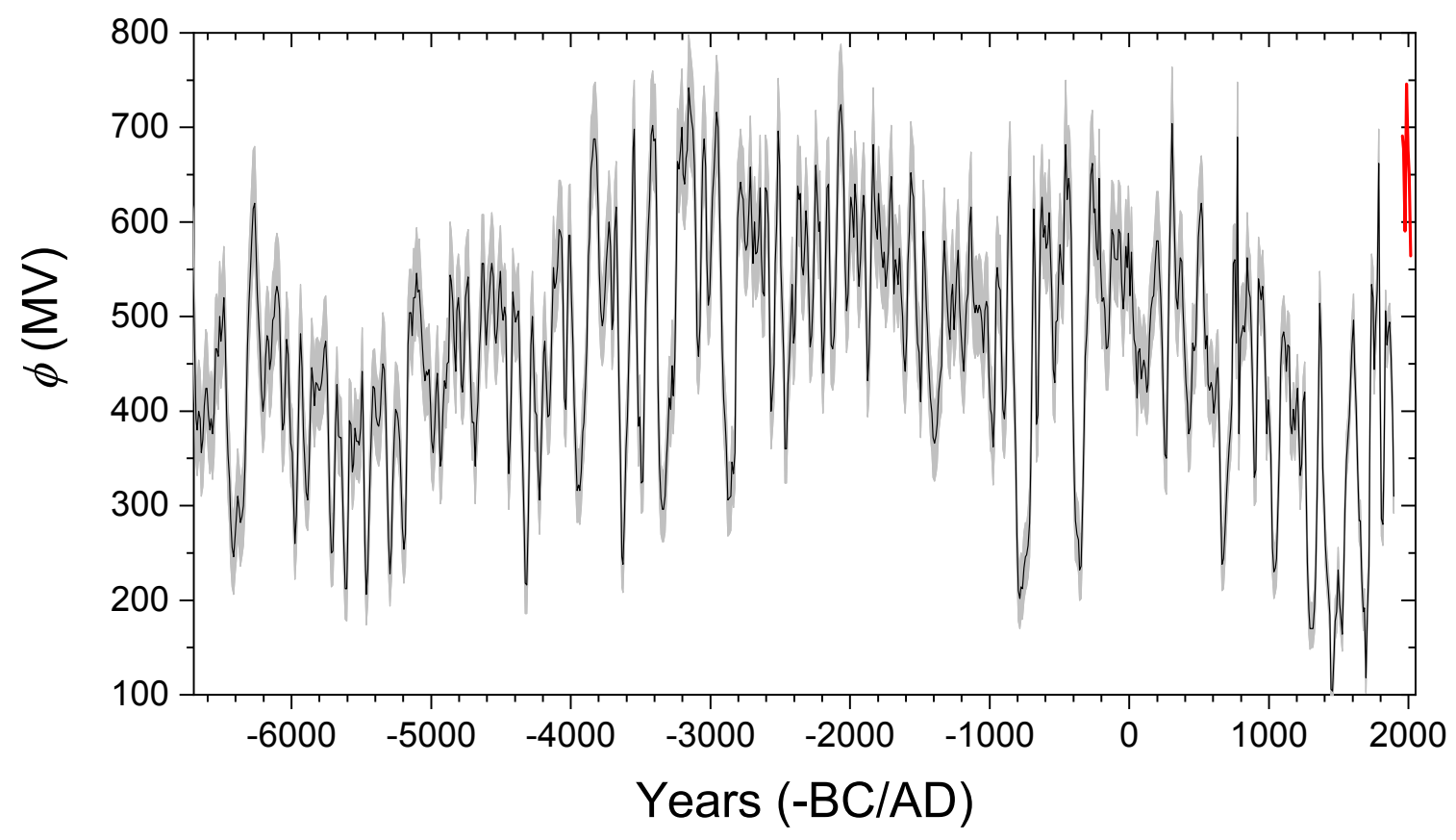

Figure 1: Multi-proxy reconstruction, based on Bayesian approach, of the decadally averaged cosmic-ray modulation potential for the mean (black curve) and $1 \sigma$ confidence interval (grey shading) for the period $6700 \mathrm{BC}-1900 \mathrm{AD}$. The red curve depicts the decadally averaged modulation potential for the instrumental era, since 1951 ([22], http://cosmicrays.oulu.fi/phi/phi.html).

1. A value of $\phi(t)$ was taken by scanning the range from $0-2000 \mathrm{MV}$.

2. An ensemble of $10^{6}$ values of the production rate of an $i$-th isotope, $Q_{i}^{\prime}$ were computed using the production model [[13], with Monte-Carlo propagation of error (model uncertainties, geomagnetic field uncertainties, and measurement uncertainties). From this ensemble, the mean $\left\langle Q_{i}{ }^{\prime}(t)\right\rangle$ and the standard deviation $\sigma_{Q, i}(t)$ were calculated.

3. The value of $\chi^{2}(\phi)=\sum_{i}\left(\frac{Q_{i}(t)-\left\langle Q_{i}{ }^{\prime}(t)\right\rangle}{\sigma_{Q, i}(t)}\right)^{2}$ was calculated as a sum over all the analyzed data series.

4. Steps 1-3 were repeated with the new value of $\phi$. The value of $\phi$ corresponding to the minimum $\chi_{\min }^{2}$ was considered as the most probable value $\phi^{*}$, and its $68 \%$ confidence level was defined as that corresponding the $\chi^{2}(\phi)=\chi_{\min }^{2}+1$.

This procedure was repeated for each moment $t$ and led to the reconstructed series of the modulation

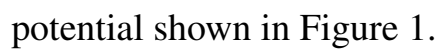

\section{Results and discussion}

The heliospheric modulation depicts a great deal of variability on different time scales as one can see in Figure m. Several features can be observed. First are the typical Grand minima of solar activity, when sunspots are nearly completely vanished and the modulation drops to a very 


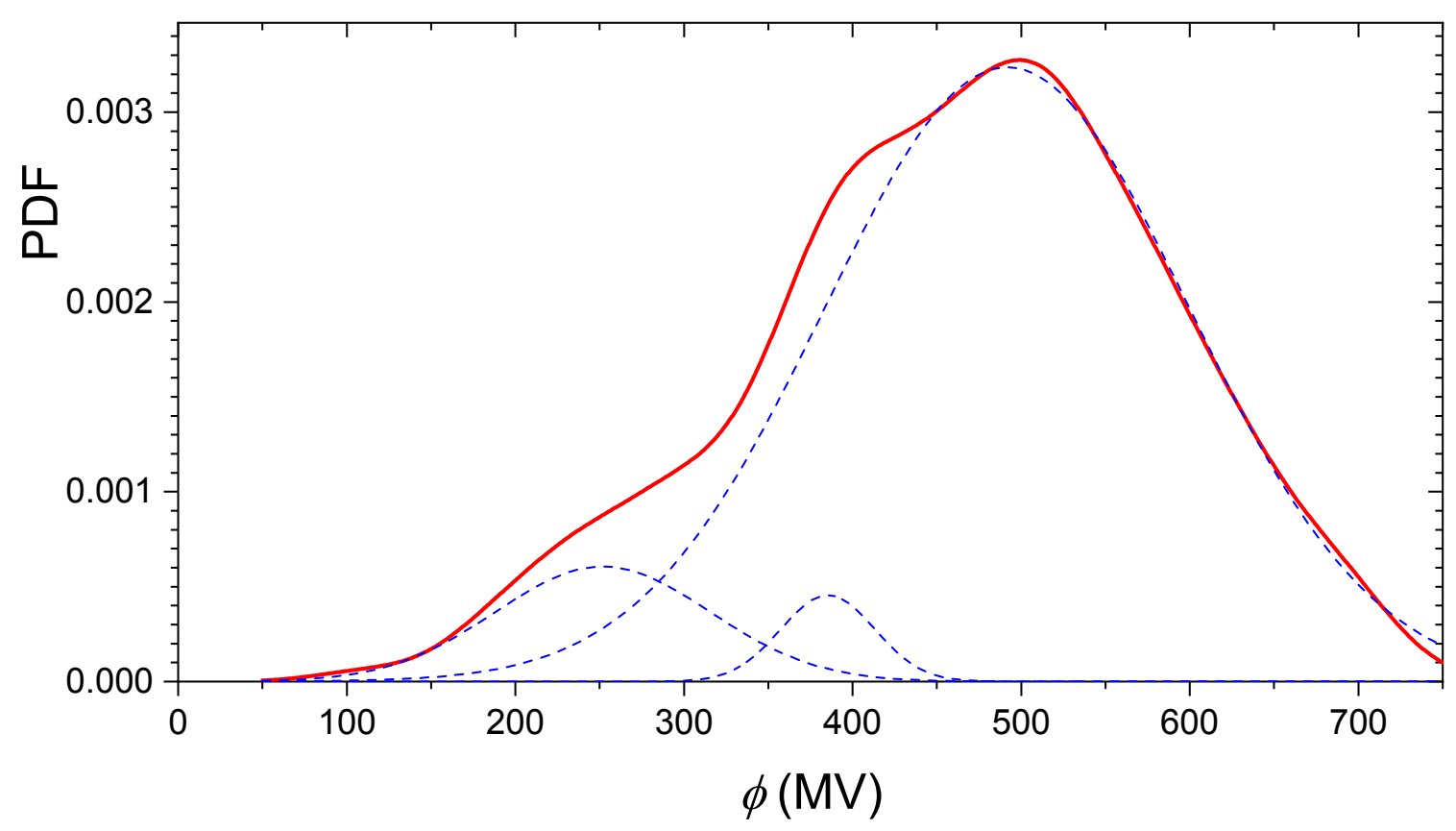

Figure 2: Kernel density estimate of the probability density function (PDF) for the occurrence probability

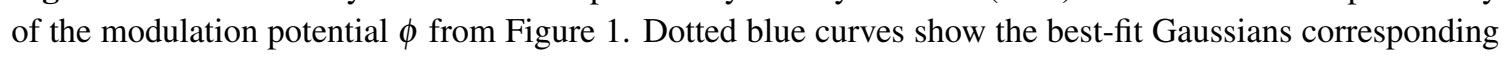
to local bumps in the distribution (maxima are at 251, 385 and $491 \mathrm{MV}$ )).

low level. This can be observed as fast and deep drops with the duration of about 100 years. Interestingly, the level of modulation during the Grand minima, is 100-200 MV throughtout the entire interval except the period between ca. $3500 \mathrm{BC}$ and $1000 \mathrm{BC}$, when the modulation drops are to the level of 300-400 MV. The latter may indicate either the absence of Grand minima over that period or some overestimate of the modulation during that period. One can also observe a long 'wave' with the lows ca. $5500 \mathrm{BC}$ and 1000-0 AD, and the high around 3000-2000 BC. The origin of this wave is unclear: it can be a real slow change of solar activity, an unknown trend in the geomagnetic data or a climate effect on the cosmogenic isotope transport in the terrestrial system [2]]. The fact that the Grand minima are not deep during the maximum of this wave, suggests that the wave likely has a terrestrial origin.

A (Gaussian) kernel density estimate of the probability density function (PDF) of the occurrence probability of the reconstructed decadal values of the modulation potential $\phi$ from Figure $\square$ is shown in Figure $\square$. The distribution can be decomposed into three Gaussians: one with the mean of $491 \mathrm{MV}$ and width $216 \mathrm{MV}$ corresponds to the normal mode of solar activity [26]; another one, with the mean $251 \mathrm{MV}$ and width $127 \mathrm{MV}$, corresponds to the special mode of Grand minima of solar activity [[23]. The third mode (mean $375 \mathrm{MV}$, width $56 \mathrm{MV}$ ) is not statistically distinguishable and most likely represents Grand minima during the maximum of the wave discussed above. A separate mode, corresponding to Grand maxima of solar activity, cannot be distinguished (cf. $[23,[26])$. 


\section{Acknowledgements}

This work was supported by the Academy of Finland (project 307411, Center of Excellence ReSoLVE).

\section{References}

[1] F. Adolphi and R. Muscheler. Synchronizing the Greenland ice core and radiocarbon timescales over the Holocene - Bayesian wiggle-matching of cosmogenic radionuclide records. Climate of the Past, 12:15-30, 2016.

[2] E. Bard, G.M. Raisbeck, F. Yiou, and J. Jouzel. Solar modulation of cosmogenic nuclide production over the last millennium: comparison between ${ }^{14} \mathrm{c}$ and ${ }^{10}$ be records. Earth Planet. Sci. Lett., 150:453-462, 1997.

[3] J. Beer, A. Blinov, G. Bonani, H.J. Hofmann, and R.C. Finkel. Use of be-10 in polar ice to trace the 11-year cycle of solar activity. Nature, 347:164-166, 1990.

[4] J. Beer, K. McCracken, and R. von Steiger. Cosmogenic Radionuclides: Theory and Applications in the Terrestrial and Space Environments. Springer, Berlin, 2012.

[5] A.-M. Berggren, J. Beer, G. Possnert, A. Aldahan, P. Kubik, M. Christl, S. J. Johnsen, J. Abreu, and B. M. Vinther. A 600 -year annual ${ }^{10}$ Be record from the NGRIP ice core, Greenland. Geophys. Res. Lett., 36:L11801, 2009.

[6] R.A. Caballero-Lopez and H. Moraal. Limitations of the force field equation to describe cosmic ray modulation. J. Geophys. Res., 109:A01101, 2004.

[7] K. Horiuchi, T. Uchida, Y. Sakamoto, A. Ohta, H. Matsuzaki, Y. Shibata, and H. Motoyama. Ice core record of 10be over the past millennium from dome fuji, antarctica: A new proxy record of past solar activity and a powerful tool for stratigraphic dating. Quat. Geochronology, 3:253-261, 2008.

[8] F. Inceoglu, R Simoniello, V. F. Knudsen, C. Karoff, J. Olsen, S. Turck-Chiéze, and B. H. Jacobsen. Grand solar minima and maxima deduced from ${ }^{10} \mathrm{be}$ and ${ }^{14} \mathrm{c}$ : magnetic dynamo configuration and polarity reversal. Astron. Astrophys., 577:A20, 2015.

[9] M.F. Knudsen and P. Riisager. Is there a link between earth's magnetic field and low-latitude precipitation? Geology, 37:71-74, 2009.

[10] K.G. McCracken, F.B. McDonald, J. Beer, G. Raisbeck, and F. Yiou. A phenomenological study of the long-term cosmic ray modulation, 850-1958 ad. J. Geophys. Res., 109(A18):12103, 2004.

[11] R. Muscheler, J. Beer, G. Wagner, C. Laj, C. Kissel, G. M. Raisbeck, F. Yiou, and P. W. Kubik. Changes in the carbon cycle during the last deglaciation as indicated by the comparison of ${ }^{10} \mathrm{Be}$ and ${ }^{14}$ C records. Earth Planet. Sci. Lett., 219:325-340, 2004.

[12] R. Muscheler, F. Joos, J. Beer, S.A. Müller, M. Vonmoos, and I. Snowball. Solar activity during the last 1000 yr inferred from radionuclide records. Quater. Sci. Rev., 26:82-97, 2007.

[13] S. V. Poluianov, G. A. Kovaltsov, A. L. Mishev, and I. G. Usoskin. Production of cosmogenic isotopes ${ }^{7} \mathrm{Be},{ }^{10} \mathrm{Be},{ }^{14} \mathrm{C},{ }^{22} \mathrm{Na}$, and ${ }^{36} \mathrm{Cl}$ in the atmosphere: Altitudinal profiles of yield functions. $J$. Geophys. Res. (Atm.), 121:8125-8136, 2016.

[14] M. Potgieter. Solar Modulation of Cosmic Rays. Living Rev. Solar Phys., 10:3, 2013. 
[15] G.M. Raisbeck, F. Yiou, J. Jouzel, and J.R. Petit. ${ }^{10}$ be and $\delta^{2} \mathrm{~h}$ in polar ice cores as a probe of the solar variability's influence on climate. Royal Soc. London Philos. Trans. Ser. A, 330:463-469, 1990.

[16] R. Roth and F. Joos. A reconstruction of radiocarbon production and total solar irradiance from the Holocene ${ }^{14} \mathrm{C}$ and $\mathrm{CO}_{2}$ records: implications of data and model uncertainties. Clim. Past, 9:1879-1909, 2013.

[17] S. K. Solanki, I. G. Usoskin, B. Kromer, M. Schüssler, and J. Beer. Unusual activity of the sun during recent decades compared to the previous 11,000 years. Nature, 431:1084-1087, 2004.

[18] F. Steinhilber, J.A. Abreu, J. Beer, I. Brunner, M. Christl, H. Fischer, U. Heikkilae, P.W. Kubik, M. Mann, K.G. McCracken, H. Miller, H. Miyahara, H. Oerter, and F. Wilhelms. 9,400 years of cosmic radiation and solar activity from ice cores and tree rings. Proc. Nat. Acad. Sci. USA, 109(16):5967-5971, 2012.

[19] I. G. Usoskin. A History of Solar Activity over Millennia. Living Rev. Solar Phys., 14:3, 2017.

[20] I. G. Usoskin, K. Alanko-Huotari, G. A. Kovaltsov, and K. Mursula. Heliospheric modulation of cosmic rays: Monthly reconstruction for 1951-2004. J. Geophys. Res., 110:A12108, 2005.

[21] I. G. Usoskin, Y. Gallet, F. Lopes, G. A. Kovaltsov, and G. Hulot. Solar activity during the Holocene: the Hallstatt cycle and its consequence for grand minima and maxim. Astron. Astrophys., 587:A150, 2016.

[22] I. G. Usoskin, A. Gil, G. A. Kovaltsov, A. L. Mishev, and V. V. Mikhailov. Heliospheric modulation of cosmic rays during the neutron monitor era: Calibration using PAMELA data for 2006-2010. $J$. Geophys. Res. (Space Phys.), 122:3875-3887, 2017.

[23] I. G. Usoskin, G. Hulot, Y. Gallet, R. Roth, A. Licht, F. Joos, G. A. Kovaltsov, E. Thébault, and A. Khokhlov. Evidence for distinct modes of solar activity. Astron. Astrophys., 562:L10, 2014.

[24] I. G. Usoskin, S. K. Solanki, and G. A. Kovaltsov. Grand minima and maxima of solar activity: new observational constraints. Astron. Astrophys., 471:301-309, 2007.

[25] M. Vonmoos, J. Beer, and R. Muscheler. Large variations in holocene solar activity: Constraints from ${ }^{10} \mathrm{be}$ in the greenland ice core project ice core. J. Geophys. Res., 111(A10):A10105, 2006.

[26] C. J. Wu, I. G. Usoskin, N. Krivova, G. A. Kovaltsov, M. Baroni, E. Bard, and S. K. Solanki. Solar activity over nine millennia: A consistent multi-proxy reconstruction. Astron. Astrophys., 615:A93, 2018.

[27] F. Yiou, G.M. Raisbeck, S. Baumgartner, J. Beer, C. Hammer, S. Johnsen, J. Jouzel, P.W. Kubik, J. Lestringuez, M. Stiévenard, M. Suter, and P. Yiou. Beryllium 10 in the greenland ice core project ice core at summit, greenland. J. Geophys. Res., 102:26783-26794, 1997. 